\title{
Mathematical modeling of rhizosphere microbial degradation with impulsive diffusion on nutrient
}

\author{
Zhong Zhao', Yanli Song ${ }^{2}$ and Liuyong Pang'
}

\section{"Correspondence:}

zhaozhong8899@163.com

'Department of Mathematics,

Huanghuai University, Zhumadian,

Henan 463000, P.R. China

\section{Springer}

\begin{abstract}
In this paper, a new mathematical modeling of rhizosphere microbial degradation with impulsive diffusion is proposed. By using the Floquet theorem, we find the rhizosphere microbe-eradication periodic solution is globally asymptotically stable if some conditions are satisfied. At the same time we also obtain the permanent conditions of the nutrients and rhizosphere microbe. Finally, a system of equations is solved by using a numerical simulation to justify our results.
\end{abstract}

MSC: $34 \mathrm{C} 05 ; 92 \mathrm{D} 25$

Keywords: rhizosphere microbial degradation; impulsive diffusion; rhizosphere microbe-eradication periodic solution; permanent

\section{Introduction}

The constructed wetland is usually used for the purpose of treating the oversupply of nutrients such as nitrogen and phosphorus in the lake. The rhizosphere microbe can play a key role in decomposing organic matter through releasing inorganic nutrient available to wetland plant. The degradation process is complex because it includes some biological and chemical reactions. Therefore, understanding of the degradation process of the rhizosphere microbe has been widely attractive to many authors [1-14]. Bunwong et al. [1] formulated a three-dimensional system of ordinary differential equations and investigated the existence of equilibria and local Hopf bifurcation. In [4], the authors compared the efficiency of a laboratory scale subsurface hybrid constructed wetland (SS-HCW) for domestic waste water treatment planted with different plants species at different hydraulic retention times. Strigul and Kravchenko [7] introduced beneficial microbes to the plant rhizosphere. They showed that the competition for limiting resources between the introduced population and the resident microorganisms was the most important factor determining PGPR survival. The authors [8] showed the exudation dynamics leading to the development of the emerging attractors and synchronized oscillations of microbial populations, carbon and oxygen concentrations. Zhao et al. [14] proposed a nonlinear mathematical model of the rhizosphere microbial degradation based on impulsive state feedback control. The sufficient conditions for existence of the positive order- 1 or order- 2 periodic solution were obtained by using the geometrical theory of the semi-continuous dynamical system.

(c) 2016 Zhao et al. This article is distributed under the terms of the Creative Commons Attribution 4.0 International License (http://creativecommons.org/licenses/by/4.0/), which permits unrestricted use, distribution, and reproduction in any medium, provided you give appropriate credit to the original author(s) and the source, provide a link to the Creative Commons license, and indicate if changes were made. 
In fact, the dispersal phenomenon is ubiquitous, which has attracted many interests of the researchers [15-18]. Jiao et al. [17] considered a five-dimensioned chemostat model with impulsive diffusion and pulse input and they obtained the stability of microorganismextinction periodic solution and permanence of the model. In [18], the authors indicated population dispersal is beneficial to pest control for some ranges of dispersal rates.

Since the rhizosphere microbial degradation is a complex process including some biological and chemical reactions, it is important to understand how this rhizosphere system operates. The mathematical model may be an important tool to understand the complex process and key parameters affecting the rhizosphere microbial degradation.

The paper is organized as follows: a mathematical model with impulsive diffusion is proposed in Section 2. In Sections 3 and 4, the stability of rhizosphere microbe eradication periodic solution and permanence are given, respectively. Finally, we give a brief discussion.

\section{Model description and preliminaries}

The rhizosphere is usually defined as a narrow zone of soil directly affected by the presence of plant root [19]. Considering the complexity of the degradation process, we suppose the rhizosphere system comprises two patches which is connected by impulsive diffusion (see Figure 1). The plant rhizosphere is directly considered as a chemostat, which is defined as patch 1 and the region outside the plant rhizosphere is called patch 2 (see Figure 1). Let $N_{1}(t)$ be the organic concentration of the region outside the rhizosphere (patch 2). $N_{2}(t)$ denotes the organic concentration of the rhizosphere (patch 1$) . N^{0}$ denotes the input concentration of the organic matter. $Q$ and $D$ are the dilution rates. The growth of the rhizosphere microbe is supposed to follow the Monod equation involving the organic concentration $N_{2}(t)$ as well as the microbial concentration $x(t)\left(i . e . \frac{\mu N_{2}(t) x(t)}{\delta\left(K+N_{2}(t)\right)}\right)$, where $\mu$ is the maximum specific growth rate and the constant $\delta$ is a yield term, $K$ is a half-saturation constant. $m$ is the mortality of the rhizosphere microbe. $d(0<d<1)$ is diffusive rate between patch 1 and patch 2 , which shows the net exchange from patch $j$ to patch $i$ is proportional to the difference $N_{j}-N_{i}$.

Figure 1 Illustration of the impulsive diffusion.

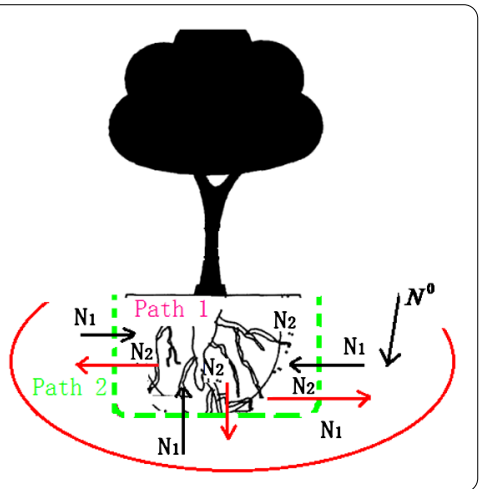


Based on the above description and [1-18], we formulate the following model:

$$
\left\{\begin{array}{l}
\frac{d N_{1}}{d t}=Q\left(N^{0}-N_{1}\right), \\
\frac{d N_{2}}{d t}=-D N_{2}-\frac{\mu N_{2} x}{\delta\left(K+N_{2}\right)}, \\
\frac{d x}{d t}=\frac{\mu N_{2} x}{K+N_{2}}-m x, \\
\Delta N_{1}=d\left(N_{2}-N_{1}\right), \\
\Delta N_{2}=d\left(N_{1}-N_{2}\right), \\
\Delta x=0,
\end{array} \quad t \neq n T,\right.
$$

where $T$ is the impulsive period, $n \in N=\{1,2,3, \ldots\}, \Delta N_{i}=N_{i}\left(t^{+}\right)-N_{i}(t)(i=1,2), \Delta x=$ $x\left(t^{+}\right)-x(t)$.

For convenience, we first give the basic properties of the following system:

$$
\begin{cases}\frac{d N_{1}}{d t}=Q\left(N^{0}-N_{1}\right), & t \neq n T, \\ \frac{d N_{2}}{d t}=-D N_{2}, & \\ \Delta N_{1}=d\left(N_{2}-N_{1}\right), & \\ \Delta N_{2}=d\left(N_{1}-N_{2}\right), & t=n T .\end{cases}
$$

For $(n T,(n+1) T]$, we solve the first two equations of system (2.2) and obtain

$$
\begin{cases}N_{1}(t)=N^{0}+\left(N_{1}\left(n T^{+}\right)-N^{0}\right) e^{-Q(t-n T)}, & n T \leq t<(n+1) T \\ N_{2}(t)=N_{2}\left(n T^{+}\right) e^{-D(t-n T)}, & n T \leq t<(n+1) T\end{cases}
$$

system (2.3) describes the nutrient concentrations of patch 1 and patch 2 for $(n T,(n+1) T]$. At the impulsive moment, system (2.2) becomes

$$
\left\{\begin{array}{l}
N_{1}(n+1) T^{+}=(1-d) N^{0}\left(1-e^{-Q T}\right)+(1-d) N_{1}\left(n T^{+}\right) e^{-Q T}+d N_{2}\left(n T^{+}\right) e^{-D T} \\
N_{2}(n+1) T^{+}=d N^{0}\left(1-e^{-Q T}\right)+(1-d) N_{2}\left(n T^{+}\right) e^{-D T}+d N_{1}\left(n T^{+}\right) e^{-Q T}
\end{array}\right.
$$

System (2.4) reflects the nutrient concentrations at the impulsive moment. The dynamical properties of systems (2.3) and (2.4) determine the dynamical behaviors of system (2.2). Obviously, system (2.4) has a fixed point

$$
\left\{\begin{array}{l}
N_{1}^{*}=\frac{N^{0}\left(1-e^{-Q T}\right)\left(1-d-(1-2 d) e^{-D T}\right)}{1-(1-d)\left(e^{-D T}+e^{-Q T T}\right)+(1-2 d) e^{-(D+Q) T}}, \\
N_{2}^{*}=\frac{d N^{0}\left(1-e^{-Q T}\right)}{1-(1-d)\left(e^{-Q T}+e^{-D T}\right)+(1-2 d) e^{-(Q+D) T}} .
\end{array}\right.
$$

Similar to the method of [20], we have the following lemma.

Lemma 2.1 System (2.2) has a unique positive T-periodic solution $\left(\bar{N}_{1}(t), \bar{N}_{2}(t)\right)$, which is globally asymptotically stable, where

$$
\left\{\begin{array}{l}
\bar{N}_{1}(t)=N^{0}+\left(N_{1}^{*}-N^{0}\right) e^{-Q(t-n T)}, \\
\bar{N}_{2}(t)=N_{2}^{*} e^{-D(t-n T)}, \\
N_{1}^{*}=\frac{N^{0}\left(1-e^{-Q T}\right)\left(1-d-(1-2 d) e^{-D T}\right)}{1-(1-d)\left(e^{-D T}+e^{-Q T}\right)+(1-2 d) e^{-(D+Q) T}}, \quad n T \leq t<(n+1) T . \\
N_{2}^{*}=\frac{d N^{0}\left(1-e^{-Q T}\right)}{1-(1-d)\left(e^{-Q T}+e^{-D T}\right)+(1-2 d) e^{-(Q+D) T}},
\end{array}\right.
$$




\section{Stability of rhizosphere microbe-eradication periodic solution}

Theorem 3.1 The rhizosphere microbe-eradication periodic solution $\left(\bar{N}_{1}^{*}(t), \bar{N}_{2}^{*}(t), 0\right)$ is globally asymptotically stable if $R<1$, where $R=\frac{\mu}{m T D} \ln \frac{K+\bar{N}_{2}^{*}}{K+N_{2}^{*} e^{-D T}}$ and $N_{2}^{*}$ is defined in (2.5).

Proof First of all, we prove the local stability of the rhizosphere microbe-eradication periodic solution $\left(\bar{N}_{1}^{*}(t), \bar{N}_{2}^{*}(t), 0\right)$. The local stability of the periodic solution $\left(\bar{N}_{1}^{*}(t), \bar{N}_{2}^{*}(t), 0\right)$ is determined by considering the small-amplitude perturbations of the solution. Define $N_{1}(t)=\bar{N}_{1}^{*}(t)+u(t), N_{2}(t)=\bar{N}_{2}^{*}(t)+v(t), x(t)=w(t)$, where $u(t), v(t)$, and $w(t)$ are small enough. We have

$$
\frac{d}{d t}\left(\begin{array}{c}
u(t) \\
v(t) \\
w(t)
\end{array}\right)=\left(\begin{array}{ccc}
-Q & 0 & 0 \\
0 & -D & \frac{\mu \bar{N}_{2}^{*}(t)}{\delta\left(K+\bar{N}_{2}^{*}(t)\right)} \\
0 & 0 & \frac{\mu \bar{N}_{2}^{*}(t)}{K+\bar{N}_{2}^{*}(t)}-m
\end{array}\right)\left(\begin{array}{c}
u(t) \\
v(t) \\
w(t)
\end{array}\right) .
$$

It is easy to obtain the fundamental solution matrix:

$$
\Phi(t)=\left(\begin{array}{ccc}
e^{-Q t} & 0 & 0 \\
0 & e^{-D t} & \frac{\mu \bar{N}_{2}^{*}(t)}{\delta\left(K+\bar{N}_{2}^{*}(t)\right)} \\
0 & 0 & e^{\int_{0}^{t}\left(\frac{\mu \bar{N}_{2}^{*}(t)}{K+N_{2}^{*}(t)}-m\right) d t}
\end{array}\right) .
$$

The linearization of the equation from the fourth to the sixth is

$$
\left(\begin{array}{l}
u\left(n T^{+}\right) \\
v\left(n T^{+}\right) \\
w\left(n T^{+}\right)
\end{array}\right)=\left(\begin{array}{ccc}
1-d & d & 0 \\
d & 1-d & 0 \\
0 & 0 & 0
\end{array}\right)\left(\begin{array}{c}
u(n T) \\
v(n T) \\
w(n T)
\end{array}\right) .
$$

Thus, the monodromy matrix of (3.1) is

$$
M^{\prime}=\left(\begin{array}{ccc}
1-d & d & 0 \\
d & 1-d & 0 \\
0 & 0 & 1
\end{array}\right) \Phi(T)
$$

Let $\lambda_{1}, \lambda_{2}, \lambda_{3}$ be eigenvalues of $M^{\prime}$. It is obvious for $\lambda_{1}=(1-d) e^{-Q T}<1, \lambda_{2}=(1-d) e^{-D T}<1$. According to Floquet theory [21], we see that the rhizosphere microbe-eradication periodic solution $\left(\bar{N}_{1}^{*}(t), \bar{N}_{2}^{*}(t), 0\right)$ is locally asymptotically stable if $\lambda_{3}=e^{\int_{0}^{T}\left(\frac{\mu \bar{N}_{2}^{*}(t)}{K+N_{2}^{*}(t)}-m\right) d t}<1$, that is, $\frac{\mu}{m T D} \ln \frac{K+\bar{N}_{2}^{*}}{K+\bar{N}_{2}^{*} e^{-D T}}<1$.

In the following, we will prove the global attraction. Choose $\varepsilon>0$ such that $\varrho=$ $\frac{\mu}{D} \ln \frac{K+\varepsilon+\bar{N}_{2}^{*}}{K+\varepsilon+\bar{N}_{2}^{*} e^{-D T}}+\frac{\mu \varepsilon}{D(K+\varepsilon)} \ln \frac{(K+\varepsilon) e^{D T}+\bar{N}_{2}^{*}}{K+\varepsilon+\bar{N}_{2}^{*}}-m T<0$.

Noticing that $\frac{d N_{2}}{d t} \leq-Q N_{2}$, we consider the following comparison system:

$$
\left\{\begin{array}{l}
\left.\begin{array}{l}
\frac{d u_{1}}{d t}=Q N^{0}-Q u_{1}, \\
\frac{d v_{1}}{d t}=-D v_{1}, \\
\Delta u_{1}=d\left(v_{1}-u_{1}\right), \\
\Delta v_{1}=d\left(u_{1}-v_{1}\right),
\end{array}\right\} \quad t \neq n T \\
t=n T
\end{array}\right.
$$


we have $N_{1}(t) \leq u_{1}(t), N_{2}(t) \leq v_{1}(t)$, and $u_{1}(t) \rightarrow \bar{N}_{1}(t), v_{1}(t) \rightarrow \bar{N}_{2}(t)$ as $t \rightarrow \infty$. Then we have

$$
N_{1}(t) \leq u_{1}(t) \leq \bar{N}_{1}(t)+\varepsilon, \quad N_{2}(t) \leq v_{1}(t) \leq \bar{N}_{2}(t)+\varepsilon
$$

for $t$ large enough. For simplicity, we suppose system (3.4) holds for all $t>0$. From the third equation of system (2.1), we obtain

$$
\frac{d x}{d t} \leq x\left(\frac{\mu\left(\bar{N}_{2}(t)+\varepsilon\right)}{K+\bar{N}_{2}(t)+\varepsilon}-m\right) .
$$

Integrating the above inequality on the interval $(n T,(n+1) T]$, we get

$$
x((n+1) T) \leq x\left(n T^{+}\right) \exp \left(\int_{n T}^{(n+1) T}\left(\frac{\mu\left(\bar{N}_{2}(t)+\varepsilon\right)}{K+\bar{N}_{2}(t)+\varepsilon}-m\right) d t\right),
$$

therefore, we have $x((n+1) T) \leq x\left(n T^{+}\right) \exp (\varrho)$, thus $x(n T) \leq x\left(0^{+}\right) \exp (n \varrho)$ and $x(n T) \rightarrow \infty$ as $t \rightarrow \infty$. Since $x(t) \leq x(n T)$, we obtain $x(t) \rightarrow 0$ as $t \rightarrow \infty$.

Next, we prove $N_{1}(t) \rightarrow \bar{N}_{1}^{*}(t)$ and $N_{2}(t) \rightarrow \bar{N}_{2}^{*}(t)$ as $t \rightarrow \infty$. There exists a $t_{0}>0$ such that $0<x(t) \leq \varepsilon$ for $t \geq t_{0}$. Therefore, we have $-D N_{2}(t)-\frac{\mu \varepsilon}{\delta} \leq \frac{d N_{2}}{d t} \leq-D N_{2}$. Hence, we get $u_{2}(t) \leq N_{1}(t) \leq u_{3}(t)$ and $v_{2}(t) \leq N_{2}(t) \leq v_{3}(t)$, where $\left(u_{2}(t), v_{2}(t)\right)$ and $\left(u_{3}(t), v_{3}(t)\right)$ are the solutions of the following two comparison systems, respectively:

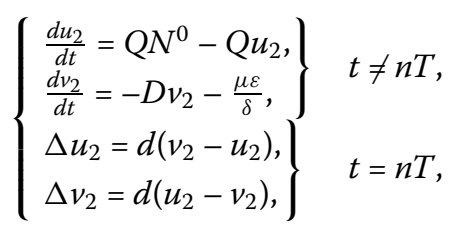

and

$$
\begin{cases}\left.\begin{array}{l}
\frac{d u_{3}}{d t}=Q N^{0}-Q u_{3}, \\
\frac{d v_{3}}{d t}=-D v_{3} \\
\Delta u_{3}=d\left(v_{3}-u_{3}\right), \\
\Delta v_{3}=d\left(u_{3}-v_{3}\right),
\end{array}\right\} \quad t \neq n T & t=n T\end{cases}
$$

The periodic solution of system (3.6) is

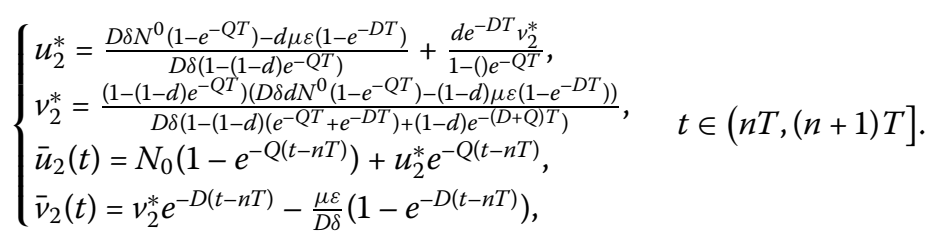

The periodic solution of system (3.7) is

$$
\left\{\begin{array}{l}
u_{3}^{*}=\frac{N^{0}\left(1-e^{-Q T}\right)\left(1-d-(1-2 d) e^{-D T}\right)}{1-(1-d)\left(e^{-D T}+e^{-Q T}\right)+(1-2 d) e^{-(D+Q) T}}, \\
v_{3}^{*}=\frac{d N^{0}\left(1-e^{-Q T}\right)}{1-(1-d)\left(e^{-Q T}+e^{-D T}\right)+(1-2 d) e^{-(Q+D) T}}, \\
\bar{u}_{3}(t)=N_{0}\left(1-e^{-Q(t-n T)}\right)+u_{3}^{*} e^{-Q(t-n T)}, \\
\bar{v}_{3}(t)=v_{3}^{*} e^{-D(t-n T)},
\end{array} \quad t \in(n T,(n+1) T] .\right.
$$


Therefore, for small enough $\varepsilon_{1}$, there exists a $t_{1}>0$ such that

$$
\bar{u}_{2}(t)-\varepsilon_{1} \leq N_{1}(t) \leq \bar{u}_{3}(t)+\varepsilon_{1}, \quad \bar{v}_{2}(t)-\varepsilon_{1} \leq N_{2}(t) \leq \bar{v}_{3}(t)+\varepsilon_{1} .
$$

Let $\varepsilon \rightarrow 0$, we get

$$
\bar{N}_{1}^{*}(t)-\varepsilon_{1} \leq N_{1}(t) \leq \bar{N}_{1}^{*}(t)+\varepsilon_{1}, \quad \bar{N}_{2}^{*}(t)-\varepsilon_{1} \leq N_{2}(t) \leq \bar{N}_{2}^{*}(t)+\varepsilon_{1}
$$

for $t$ large enough, which shows $N_{1}(t) \rightarrow \bar{N}_{1}^{*}(t)$ and $N_{2}(t) \rightarrow \bar{N}_{2}^{*}(t)$ as $t \rightarrow \infty$. The proof is completed.

\section{Permanent}

First of all, we show that all solutions of (2.1) are ultimately bounded.

Lemma 4.1 The system (2.1) is ultimately bounded.

Proof Define a function $V(t)=N_{1}(t)+N_{2}(t)+\frac{x(t)}{\delta}$. When $t \neq n T$, we have $\frac{d V}{d t}=Q N^{0}-$ $Q N_{1}-D N_{2}-\frac{m x(t)}{\delta} \leq Q N^{0}-\rho V(t)$, where $\rho=\min \{Q, D, m\}$. When $t=n T$, we also obtain $V\left(n T^{+}\right)=V(n T)$. For $(n T,(n+1) T]$, we have $V(t) \leq V(0) e^{-\rho t}+\frac{Q N^{0}}{\rho}\left(1-e^{-\rho t}\right) \rightarrow \frac{Q N^{0}}{\rho}$, as $t \rightarrow \infty$. So we have $N_{1}(t) \leq M, N_{2}(t) \leq M, x(t) \leq M$, where $\frac{Q N^{0}}{\rho} \triangleq M$.

Theorem 4.2 System (2.1) is permanent if $R>1$, where $R$ is defined in Theorem 3.1.

Proof Suppose $\left(N_{1}(t), N_{2}(t), x(t)\right)$ is a solution of (2.1) with positive initial value. From Lemma 4.1 $N_{1}(t) \leq \frac{Q N^{0}}{\rho} \triangleq M, N_{2}(t) \leq \frac{Q N^{0}}{\rho} \triangleq M, x(t) \leq \frac{Q N^{0}}{\rho} \triangleq M, t \geq 0$, we get $\frac{d N_{2}}{d t} \geq$ $-D N_{2}-\frac{\mu M}{\delta}$.

Considering the comparison system:

$$
\begin{cases}\frac{d u_{4}}{d t}=Q N^{0}-Q u_{4}, & \\ \frac{d v_{4}}{d t}=-D v_{4}-\frac{\mu M}{\delta}, & \\ \Delta u_{4}=d\left(v_{4}-u_{4}\right), & \\ \Delta v_{4}=d\left(u_{4}-v_{4}\right), & \end{cases}
$$

Similar to system (2.2), the periodic solution $\left(\bar{u}_{4}(t), \bar{v}_{4}(t)\right)$ can be given:

$$
\left\{\begin{array}{l}
u_{4}^{*}=\frac{(1-d) N^{0}-d \frac{\mu M}{D \delta}\left(1-e^{-D T}\right)}{1-(1-d) e^{-Q T}}+\frac{d e^{-D T} v_{4}^{*}}{1-(1-d) e^{-Q}}, \\
v_{4}^{*}=\frac{\left[-(1-d) \frac{\mu M}{D \delta}\left(1-e^{-D T}\right)+d N^{0}\left(1-e^{-Q T}\right)\right]\left(1-(1-d) e^{-Q T}\right)}{1-(1-d)\left(e^{-Q T}+e^{-D T}\right)+(1-2 d) e^{-(Q+D) T}}, \\
\bar{u}_{4}(t)=N_{0}\left(1-e^{-Q(t-n T)}\right)+u_{4}^{*} e^{-Q(t-n T)}, \\
\bar{v}_{4}(t)=-\frac{\mu M}{D \delta}+\left(v_{4}^{*}+\frac{\mu M}{D \delta}\right) e^{-D(t-n T)},
\end{array}\right.
$$

which is globally asymptotically stable. Hence, there exists a $\varepsilon_{2}>0$ such that $N_{1}(t) \geq$ $u_{4}(t) \geq u_{4}^{*}(t)-\varepsilon_{2} \geq u_{4}^{*}-\varepsilon_{2} \triangleq m_{1}, N_{2}(t) \geq v_{4}(t) \geq v_{4}^{*}(t)-\varepsilon_{2} \geq v_{4}^{*}-\varepsilon_{2} \triangleq m_{2}$ for $t$ large enough. In the following, we want to find $m_{3}$ such that $x(t) \geq m_{3}$ for $t$ large enough. We shall do it in the following two steps. 
Step I: Let $m_{3}>0$ and $\varepsilon_{3}$ be small enough such that

$$
\begin{aligned}
\rho= & \frac{\mu \varepsilon_{3}+\frac{\mu^{2} m_{3}}{D \delta}}{D\left(K-\varepsilon_{3}-\frac{\mu m_{3}}{D \delta}\right)} \ln \frac{K-\varepsilon_{3}+v_{5}^{*}}{\left(K-\varepsilon_{3}-\frac{\mu m_{3}}{D \delta}\right) e^{D T}+v_{5}^{*}+\frac{\mu m_{3}}{D \delta}} \\
& +\frac{1}{D} \ln \frac{K-\varepsilon_{3}-\frac{\mu m_{3}}{D \delta}+\left(v_{5}^{*}+\frac{\mu m_{3}}{D \delta}\right) e^{-D T}}{K-\varepsilon_{3}+v_{5}^{*}}-m T>0 .
\end{aligned}
$$

We will prove $x_{2}(t)<m_{3}$ cannot hold for all $t \geq 0$. Otherwise, $\frac{d N_{2}}{d t} \geq-D N_{2}-\frac{\mu m_{3}}{\delta}$, we consider the following comparison system:

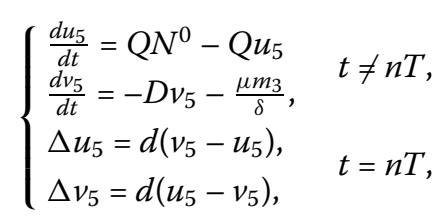

we get $N_{1}(t) \geq u_{5}(t), N_{2}(t) \geq v_{5}(t)$, and $u_{5}(t) \rightarrow \bar{u}_{5}^{*}(t), v_{5}(t) \rightarrow \bar{v}_{5}^{*}(t)$ as $t \rightarrow \infty$, where $\left(\bar{u}_{5}^{*}(t), \bar{v}_{5}^{*}(t)\right)$ is the periodic solution of system $(4.3)$ and $\left(\bar{u}_{5}^{*}(t), \bar{v}_{5}^{*}(t)\right)$ is given as

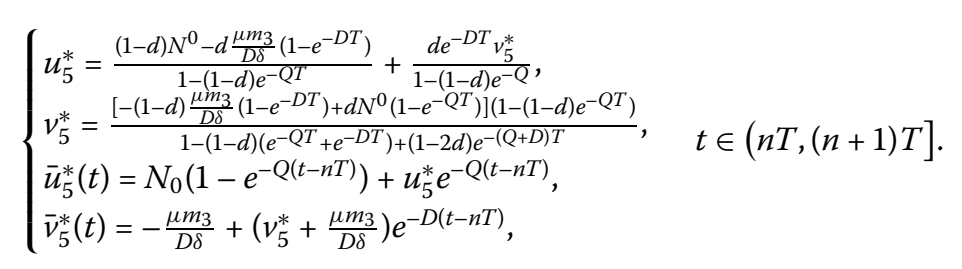

Hence for $\varepsilon_{3}$ small enough, there exists a $T_{1}>0$ such that $N_{1}(t) \geq u_{5}^{*}(t)-\varepsilon_{3}, N_{2}(t) \geq v_{5}^{*}(t)-$ $\varepsilon_{3}$, and

$$
\frac{d x}{d t} \geq\left(\frac{\mu\left(v_{5}^{*}(t)-\varepsilon_{3}\right)}{K+v_{5}^{*}(t)-\varepsilon_{3}}-m\right) x, \quad t \geq T_{1} .
$$

Let $n_{1} \in N$ and $n_{1} T>T_{1}$, integrating (4.5) on the interval $(n T,(n+1) T], n>n_{1}$, we obtain

$$
x((n+1) T) \geq x\left(n T^{+}\right) \exp \left(\int_{n T}^{(n+1) T}\left(\frac{\mu\left(v_{5}^{*}(t)-\varepsilon_{3}\right)}{K+v_{5}^{*}(t)-\varepsilon_{3}}-m\right) d t\right)=x\left(n T^{+}\right) \exp (\rho) .
$$

Then $x\left(\left(n_{1}+k\right) T\right) \geq x\left(n_{1} T\right) \exp (k \rho) \rightarrow \infty$ as $k \rightarrow \infty$, which is in contradiction to the boundedness of $x(t)$. Therefore, there is a $t_{1}>0$ such that $x\left(t_{1}\right)>m_{3}$. If $x(t) \geq m_{3}$ for all $t>t_{1}$, then our aim is obtained. Otherwise, there exists a $\bar{t}_{1}>t_{1}$ such that $x\left(\bar{t}_{1}\right)<m_{3}$. Setting $t^{*}=\inf _{t>t^{*}}\left\{x_{2}(t)<m_{3}\right\}$, then we have $x_{2}(t) \geq m_{3}$ for $t \in\left[t, t^{*}\right)$, and $x_{2}\left(t^{*}\right)=m_{3}$.

Steps II: Since $x(t)$ is continuous, suppose $t^{*} \in\left(n_{1} T,\left(n_{1}+1\right) T\right], n_{1} \in N$, select $n_{2} \in N$, $n_{3} \in N$, such that

$$
\begin{aligned}
& n_{2} T>\min \left\{\frac{1}{Q} \frac{M+u_{5}^{*}}{\varepsilon_{3}}, \frac{1}{D} \frac{M+v_{5}^{*}}{\varepsilon_{3}}\right\}, \\
& \exp \left(\eta\left(n_{2}+1\right) T\right) \exp \left(n_{3} \rho\right)>1,
\end{aligned}
$$

where $\eta=\frac{\mu \Phi}{K+\Phi}-m<0$, where $\Phi=-\frac{\mu m_{3}}{D \delta}+\left(v_{5}^{*}+\frac{\mu m_{3}}{D \delta}\right) e^{-D T}$ and $v_{5}^{*}$ is defined in (4.4). 

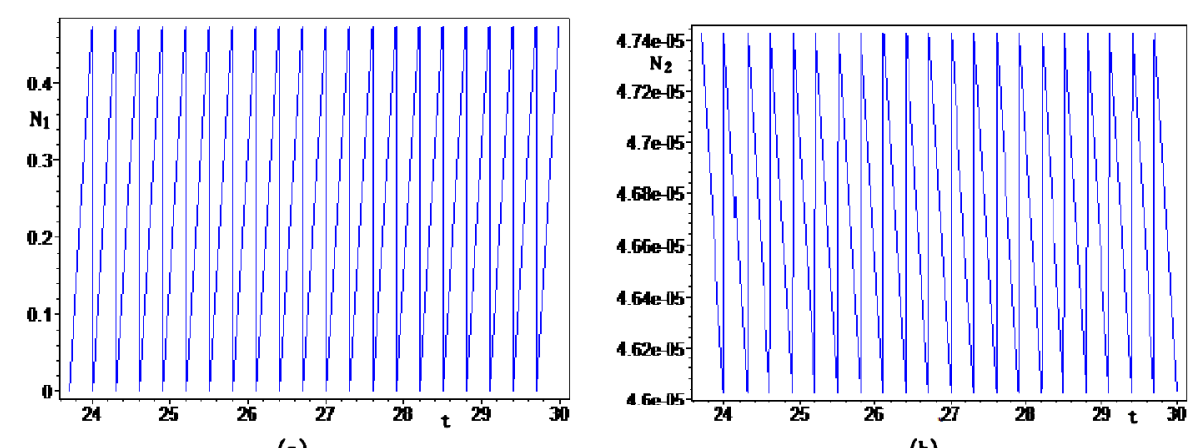

(a)

(b)
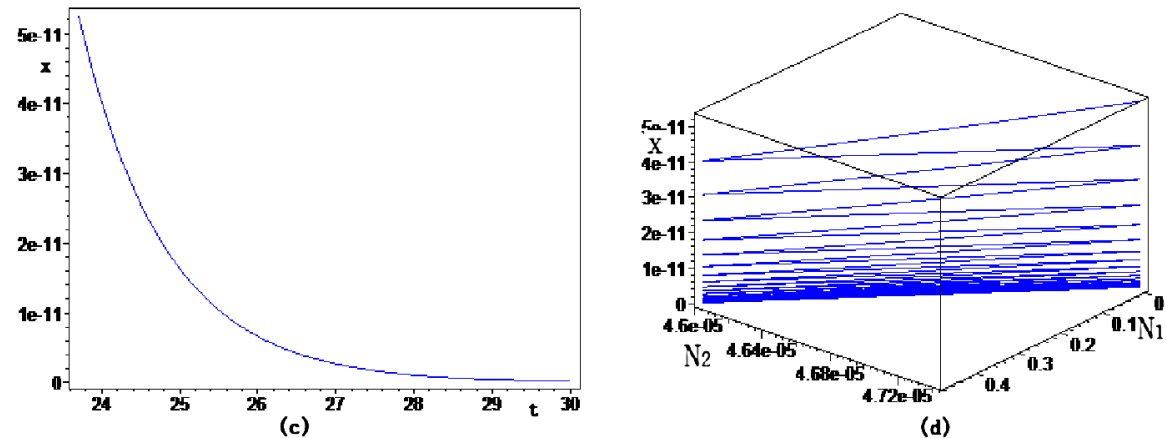

(d)

Figure 2 Dynamical behavior of the system (2.1) with $Q=0.08, N^{0}=20, D=0.1, \mu=0.1, \delta=0.5$, $K=1.8, m=0.9, d=0.0001, N_{1}(0)=18, N_{2}(0)=0.2, x(0)=0.1, R=0.39<1$. (a) Time-series of the nutrient concentration outside the rhizosphere (patch 2). (b) Time-series of the nutrient concentration inside the rhizosphere (patch 1). (c) Phase portrait denoting the rhizosphere microbe-eradication periodic solution $\left(\bar{N}_{1}^{*}(t), \bar{N}_{2}^{*}(t), 0\right)$ is globally asymptotically stable for $R<1$.

Let $T^{\prime}=n_{2} T+n_{3} T$, we claim that there must exist a $t^{\prime} \in\left(\left(n_{1}+1\right) T,\left(n_{1}+1\right) T+T^{\prime}\right]$ such that $x(t) \geq m_{3}$, otherwise $x(t)<m_{3}$ for $t \in\left(\left(n_{1}+1\right) T,\left(n_{1}+1\right) T+T^{\prime}\right]$.

Considering (4.3) with

$$
u_{5}\left(\left(n_{1}+1\right) T^{+}\right)=N_{1}\left(\left(n_{1}+1\right) T^{+}\right), \quad v_{5}\left(\left(n_{1}+1\right) T^{+}\right)=N_{2}\left(\left(n_{1}+1\right) T^{+}\right)
$$

we have

$$
\begin{aligned}
& u_{5}(t)=\left(u_{5}\left(\left(n_{1}+1\right) T^{+}\right)-u_{5}^{*}\right) e^{-Q\left(t-\left(n_{1}+1\right) T\right)}+\bar{u}_{5}^{*}(t), \\
& v_{5}(t)=\left(v_{5}\left(\left(n_{1}+1\right) T^{+}\right)-v_{5}^{*}\right) e^{-D\left(t-\left(n_{1}+1\right) T\right)}+\bar{v}_{5}^{*}(t)
\end{aligned}
$$

for $t \in(n T,(n+1) T], n_{1}+1<n \leq n_{1}+n_{2}+n_{3}+1$. Then

$$
\begin{aligned}
& \left|u_{5}(t)-\bar{u}_{5}^{*}(t)\right| \leq\left(M+u_{5}^{*}\right) e^{-Q\left(t-\left(n_{1}+1\right) T\right)}<\varepsilon_{3}, \\
& \left|v_{5}(t)-\bar{v}_{5}^{*}(t)\right| \leq\left(M+v_{5}^{*}\right) e^{-D\left(t-\left(n_{1}+1\right) T\right)}<\varepsilon_{3},
\end{aligned}
$$

we have $u(t) \leq \bar{u}^{*}(t)+\varepsilon_{3}, v(t) \leq \bar{v}^{*}(t)+\varepsilon_{3}$ for $\left(n_{1}+1+n_{2}\right) T \leq t \leq\left(n_{1}+1\right) T+T^{\prime}$, which shows system (4.5) holds. As in step I, we have $x\left(\left(n_{1}+n_{2}+n_{3}+1\right) T\right) \geq x\left(\left(n_{1}+n_{2}+1\right) T\right) \exp \left(n_{3} \rho\right)$. 


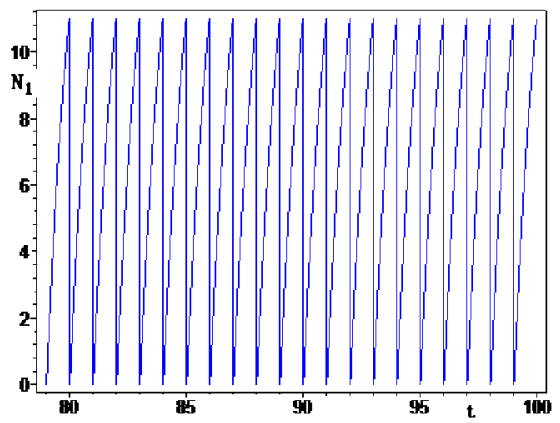

(a)

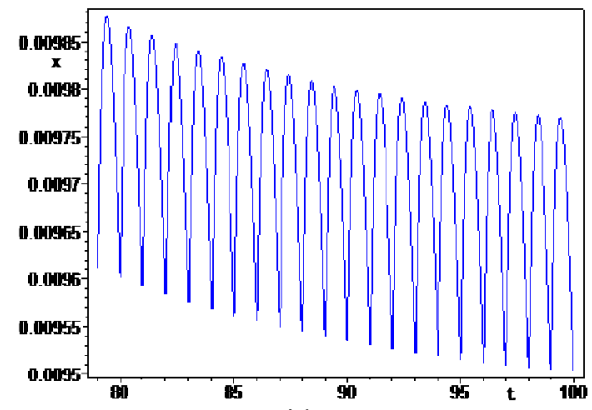

(c)

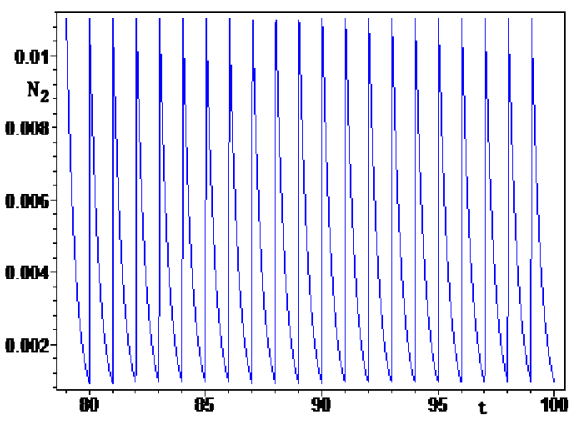

(b)

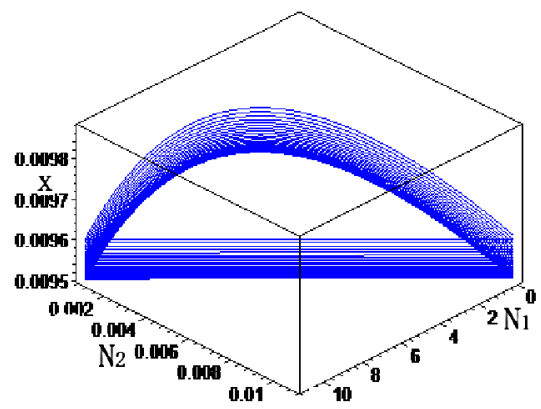

(d)

Figure 3 Dynamical behavior of the system (2.1) with $Q=0.8, N^{0}=20, D=0.1, \mu=0.3, \delta=0.1$, $K=1.8, m=0.1, d=0.0001, N_{1}(0)=18, N_{2}(0)=0.2, x(0)=0.1, R=15.99>1$. (a) Time-series of the nutrient concentration outside the rhizosphere (patch 2). (b) Time-series of the nutrient concentration inside the rhizosphere (patch 1). (c) Phase portrait implying system (2.1) is permanent for $R>1$.

From system (2.1), we have

$$
\frac{d x}{d t} \geq\left(\frac{\mu \Phi}{K+\Phi}-m\right) x(t)=\eta x(t)
$$

Integrating the above equation on $\left(t^{*},\left(n_{1}+1+n_{2}\right) T\right]$, we obtain $x\left(\left(n_{1}+1+n_{2}\right) T\right) \geq$ $m_{3} e^{\eta\left(n_{2}+1\right) T}$, then

$$
x\left(\left(n_{1}+1+n_{2}+N_{3}\right) T\right) \geq m_{3} e^{\eta\left(n_{2}+1\right) T} e^{n_{3} T}>m_{3},
$$

which is a contradiction. Let $\bar{t}=\inf _{t \geq t^{*}}\left\{x(t) \geq m_{3}\right\}$, thus $x(\bar{t}) \geq m_{3}$ for $t \in\left[t^{*}, \bar{t}\right]$, we get $x(t) \geq x\left(t^{*}\right) e^{\eta\left(t-t^{*}\right)} \geq m_{3} e^{\eta\left(n_{1}+1+n_{2}\right) T} \triangleq \bar{m}_{3}$ for $t \geq \bar{t}$. The same arguments can be continued since $x(\bar{t}) \geq m_{3}$. Hence $x(t) \geq \bar{m}_{3}$ for $t \geq t_{1}$.

\section{Discussion}

Since the rhizosphere microbial degradation undergoes a series of complex biochemical reactions, the degradation process of rhizosphere microbe may be affected by many factors. It is unrealistic to expect the existing model predicts all possible results of microbial degradation, therefore, each of the models has some validity and application limits [7]. In this paper, we have formulated a mathematical modeling of rhizosphere microbial degradation with impulsive diffusion and obtained the rhizosphere microbe-eradication periodic solution $\left(\bar{N}_{1}^{*}(t), \bar{N}_{2}^{*}(t), 0\right)$ is globally asymptotically stable for $R<1$, which is showed in 
Figure 2 with the parameters $Q=0.08, N^{0}=20, D=0.1, \mu=0.1, \delta=0.5, K=1.8, m=0.9$, $d=0.0001, N_{1}(0)=18, N_{2}(0)=0.2, x(0)=0.1, R=0.39<1$. We can see the variables $N_{1}(t)$, $N_{2}(t)$ oscillate in a stable periodical cycle. On the contrary, $x(t)$ rapidly decreases to zero. From Theorem 4.2, we also have proved system (2.1) is permanent for $R>1$, which is simulated in Figure 3 with the parameters $Q=0.8, N^{0}=20, D=0.1, \mu=0.3, \delta=0.1, K=1.8$, $m=0.1, d=0.0001, N_{1}(0)=18, N_{2}(0)=0.2, x(0)=0.1, R=15.99>1$. The variables $N_{1}(t)$, $N_{2}(t)$ and $x(t)$ oscillate in a stable periodical cycle, respectively.

\section{Competing interests}

The authors declare that they have no competing interests.

\section{Authors' contributions}

ZZ formulated the mathematical modeling of rhizosphere microbial degradation and carried out the analysis. YS gave some constructive comments. LP corrected the manuscript. All authors have read and approved the final manuscript.

\section{Author details}

${ }^{1}$ Department of Mathematics, Huanghuai University, Zhumadian, Henan 463000, P.R. China. ${ }^{2}$ Department of Economic Management, Henan College of Quality Engineering Vocation, Pingdingshan, Henan 467001, P.R. China.

\section{Acknowledgements}

We would like to express our sincere appreciation to the anonymous reviewers for their valuable and constructive suggestions. This work is supported by the National Natural Science Foundation of China (No. 11371164), NSFC-Talent Training Fund of Henan (U1304104) and Innovative talents of science and technology plan in Henan province (15HASTIT014) and the young backbone teachers of Henan (No. 2013GGJS-214).

Received: 11 August 2015 Accepted: 8 December 2015 Published online: 26 January 2016

\section{References}

1. Bunwong, K, Sae-jie, W, Lenbury, Y: Modelling nitrogen dynamics of a constructed wetland: nutrient removal process with variable yield. Nonlinear Anal. 71, 1538-1546 (2009)

2. Kuzyakov, $Y, X u, X$ : Competition between roots and microorganisms for nitrogen: mechanisms and ecological relevance. New Phytol. 198, 656-669 (2013)

3. Peng, J, Wu, G, Chen, K: Studies on identification of a wetland plant rhizosphere microorganism LF2, optimization of the fermental conditions and application effect in constructed wetland. Master's thesis, Central China Normal University (2011)

4. Sehar, S, Sumera, Naeem, S, Perveen, I, Ali, N, Ahmed, S: A comparative study of macrophytes influence on wastewater treatment through subsurface flow hybrid constructed wetland. Ecol. Eng. 81, 62-69 (2015)

5. Khan, AG: Role of soil microbes in the rhizospheres of plants growing on trace metal contaminated soils in phytoremediation. J. Trace Elem. Med. Biol. 18, 355-364 (2005)

6. Shukla, JB, Misra, AK, Chandra, P: Mathematical modeling and analysis of the depletion of dissolved oxygen in eutrophied water bodies affected by organic pollutants. Nonlinear Anal., Real World Appl. 9, 1851-1865 (2008)

7. Strigul, NS, Kravchenko, LV: Mathematical modeling of PGPR inoculation into the rhizosphere. Environ. Model. Softw. 21, 1158-1171 (2006)

8. Beckett, PM, Armstrong, W, Armstrong, J: Mathematical modelling of methane transport by Phragmites: the potential for diffusion within the roots and rhizosphere. Aquat. Bot. 69, 293-312 (2001)

9. Atangana, A, Alkahtani, BST: Analysis of the Keller-Segel model with a fractional derivative without singular kernel. Entropy 17, 4439-4453 (2015)

10. Atangana, A, Alkahtani, BST: Modeling the spread of rubella disease using the concept of with local derivative with fractional parameter: beta-derivative. Complexity (2015). doi:10.1002/cplx.21704

11. Faybishenko, B, Molz, F: Nonlinear rhizosphere dynamics yields synchronized oscillation of microbial populations, carbon and oxygen concentrations induced by root exudation. Proc. Environ. Sci. 19, 369-378 (2013)

12. Atangana, A, Doungmo Goufo, EF: On the mathematical analysis of Ebola hemorrhagic fever: deathly infection disease in West African countries. BioMed Res. Int. 2014, Article ID 261383 (2014)

13. Atangana, A, Oukouomi Noutchie, SC: Model of break-bone fever via beta-derivatives. BioMed Res. Int. 2014, Article ID 523159 (2014)

14. Zhao, H, Huang, X, Zhang, X: Hopf bifurcation and harvesting control of a bioeconomic plankton model with delay and diffusion terms. Physica A 421, 300-315 (2015)

15. Zhao, Z, Pang, L, Zhao, Z, Luo, C: Impulsive state feedback control of the rhizosphere microbial degradation in the wetland plant. Discrete Dyn. Nat. Soc. 2015, Article ID 612354 (2015)

16. Dhar, J, Jatav, KS: Mathematical analysis of a delayed stage-structured predator-prey model with impulsive diffusion between two predators territories. Ecol. Complex. 16, 59-67 (2013)

17. Jiao, J, Ye, K, Chen, L: Dynamical analysis of a five-dimensioned chemostat model with impulsive diffusion and pulse input environmental toxicant. Chaos Solitons Fractals 44, 17-27 (2011)

18. Yang, J, Tang, S: Effects of population dispersal and impulsive control tactics on pest management. Nonlinear Anal. Hybrid Syst. 3, 487-500 (2009)

19. Philippot, L, Raaijmakers, JM, Lemanceau, P, van der Putten, WH: Going back to the roots: the microbial ecology of the rhizosphere. Nat. Rev. Microbiol. 11, 789-799 (2013). doi:10.1038/nrmicro3109 
20. Zhao, Z, Zhang, X, Chen, L: The effect of pulsed harvesting policy on the inshore-offshore fishery model with the impulsive diffusion. Nonlinear Dyn. 63, 537-545 (2011)

21. Bainov, D, Simeonov, P: Impulsive Differential Equations: Periodic Solutions and Applications. Pitman Monographs and Surveys in Pure and Applied Mathematics, vol. 66 (1993)

Submit your manuscript to a SpringerOpen ${ }^{\circ}$ journal and benefit from:

- Convenient online submission

Rigorous peer review

- Immediate publication on acceptance

- Open access: articles freely available online

- High visibility within the field

- Retaining the copyright to your article

Submit your next manuscript at $\boldsymbol{s p r i n g e r o p e n . c o m ~}$ 